\title{
Shape Transitions and Chiral Symmetry Breaking in the Energy Landscape of the Mitotic Chromosome
}

\author{
Bin Zhang ${ }^{1,2}$ and Peter G. Wolynes, ${ }^{1,2,3, *}$ \\ ${ }^{1}$ Department of Chemistry, Rice University, Houston, Texas 77005, USA \\ ${ }^{2}$ Center for Theoretical Biological Physics, Rice University, Houston, Texas 77005, USA \\ ${ }^{3}$ Department of Physics and Astronomy, Rice University, Houston, Texas 77005, USA
}

(Received 10 November 2015; published 14 June 2016)

\begin{abstract}
We derive an unbiased information theoretic energy landscape for chromosomes at metaphase using a maximum entropy approach that accurately reproduces the details of the experimentally measured pairwise contact probabilities between genomic loci. Dynamical simulations using this landscape lead to cylindrical, helically twisted structures reflecting liquid crystalline order. These structures are similar to those arising from a generic ideal homogenized chromosome energy landscape. The helical twist can be either right or left handed so chiral symmetry is broken spontaneously. The ideal chromosome landscape when augmented by interactions like those leading to topologically associating domain formation in the interphase chromosome reproduces these behaviors. The phase diagram of this landscape shows that the helical fiber order and the cylindrical shape persist at temperatures above the onset of chiral symmetry breaking, which is limited by the topologically associating domain interaction strength.
\end{abstract}

DOI: 10.1103/PhysRevLett.116.248101

When cells divide, their chromosomes dramatically condense before forming a pair of sister chromatids that exhibits the famous $X$ shape of the mitotic phase. Microscopy reveals the overall morphology of the mitotic chromatin, but its internal structure remains controversial [1-3]. Numerous structural models of the mitotic chromosome have been proposed, including the radial loop model [3], the chromatin network model [1], and the hierarchical folding model [2], and a consensus picture has yet to emerge.

A complete understanding of chromosomal organization is challenging since the condensation of the chromosome into its dense mitotic form permits many different orderings and phase transitions. Furthermore, owing to the large scale of the chromosome, nonequilibrium glassy dynamics and possible kinetic control of structure formation may arise as well [4-13]. In this Letter, we derive information theoretic energy landscapes for the mitotic chromosome following the maximum entropy approach developed in our previous manuscript [4]. As shown by us and many others, the effective equilibrium picture provided by maximum entropy models can be useful to provide biological insights into many nonequilibrium systems such as protein sequence evolution [14], gene regulatory networks [15], and neuronal networks [16]. The information theoretic energy landscapes for the mitotic chromosome reproduce contact frequencies between specific pairs of genomic loci inside the cell nucleus, as measured by genome-wide chromosome conformation capture (Hi-C) via chemical cross-linking [7]. Simulation of these landscapes yields ensembles of high-resolution three-dimensional conformations that reveal the structural organization of the mitotic chromosome. Theoretical analysis of these landscapes uncovers novel phenomena unique to the mitotic chromosome, and provides insight on the chromosome condensation pathway from interphase to metaphase. In particular, we use these landscapes to investigate the determinants of the overall chromosome shape, along with the liquid crystalline and chiral ordering by computing the chromosome's phase diagram.

As shown in our previous manuscript on the interphase chromosome [4], the energy landscape that reproduces Hi-C contact probabilities while simultaneously maximizing the information entropy adopts the following form:

$$
U_{\mathrm{ME}}(r)=U(r)+\sum_{i j} \alpha_{i j} f\left(r_{i j}\right) .
$$

$U(r)$ denotes the potential energy function of a connected homopolymer modeled as beads on a string, with each bead presenting a 100-kilo base pair long genomic segment [see the Supplemental Material [17] for the definition of $U(r)$ ]. We note the modeling scale at $100 \mathrm{~Kb}$ is mostly constrained by the resolution of the Hi-C performed in Ref. [7], but it is also consistent with a $30 \mathrm{~nm}$ fiber description of the chromosome [17]. $f\left(r_{i j}\right)$ monitors contact formation, and represents the probability that two polymers whose centers are separated by a distance $r_{i j}$ will form a detectable cross-link. We take $f\left(r_{i j}\right)$ to have the form of a switching function inspired by polymer physics for each bead. The set of $\left\{\alpha_{i j}\right\}$ determines the strength of the contact interactions between specific genomic loci. The $\boldsymbol{\alpha}$ values are found by minimizing the objective function $\Gamma(\boldsymbol{\alpha})$ defined as

$$
\Gamma(\boldsymbol{\alpha})=\ln \frac{Z(\boldsymbol{\alpha})}{Z_{o}}+\beta \sum_{i j} \alpha_{i j} f_{i j}^{\exp },
$$


where $\beta$ is the inverse information theoretic temperature. $Z(\boldsymbol{\alpha})$ and $Z_{o}$ are the partition functions for $U_{\mathrm{ME}}(r)$ and $U(r)$, respectively. Since $Z(\boldsymbol{\alpha})$ is the partition function for the maximum entropy Hamiltonian $U_{\mathrm{ME}}(r)$ with parameters $\left\{\alpha_{i j}\right\}, \Gamma(\boldsymbol{\alpha})$ is the loss of information theoretic entropy of an ensemble owing to its being biased to reproduce the input data, having only made the prior assumption that the chromosome is a connected polymer chain with partition function $Z_{o}$. Using a cumulant expansion, $\Gamma(\boldsymbol{\alpha})$ can be approximated as $\left(\beta^{2} / 2\right) \boldsymbol{\alpha}^{T} \boldsymbol{B} \boldsymbol{\alpha}-\beta\left[\langle\boldsymbol{f}\rangle-\boldsymbol{f}^{\exp }\right]^{T} \boldsymbol{\alpha}$, where $\boldsymbol{B}=$ $\left\langle\boldsymbol{f} \boldsymbol{f}^{T}\right\rangle-\langle\boldsymbol{f}\rangle\left\langle\boldsymbol{f}^{T}\right\rangle[26] . \boldsymbol{f}$ and $\boldsymbol{f}^{\text {exp }}$ are column vectors of contact probabilities for all the pairs of genomic loci included in Eq. (1) determined from the simulation and experimental measurements, respectively. The approximate expression for $\Gamma(\boldsymbol{\alpha})$ takes its extreme value at $\boldsymbol{\alpha}=(1 / \beta) \boldsymbol{B}^{-1}\left[\langle\boldsymbol{f}\rangle-\boldsymbol{f}^{\text {exp }}\right]$. Since this expression is only an approximate solution for $\boldsymbol{\alpha}$, as outlined in Ref. [4], we iterate the procedure to determine final values for $\boldsymbol{\alpha}$ for which the contact probabilities determined from simulation agree as closely as possible with the experimental input. A schematic representation of the Hamiltonian from direct inversion is provided in the top panel of Fig. 1(c). Because the $\left\{\alpha_{i j}\right\}$ are allowed to vary independently, the interactions between different loci adopt distinct values indicated with different colors.

The inferred "agnostic" information theoretic energy landscape reproduces the experimental contact probabilities as seen in Fig. 1, which compares the experimental and simulated contact probability maps shown in the upper and the lower triangle, respectively. As seen in Fig. S1(a) [17], the individual pair probabilities obtained by simulating the landscape are very accurately reproduced with a very high $R^{2} \sim 0.93$.

While using only probabilistic contact pair input alone, the inferred ensemble of structures immediately reproduces the large scale shape and size of chromosome as seen in the
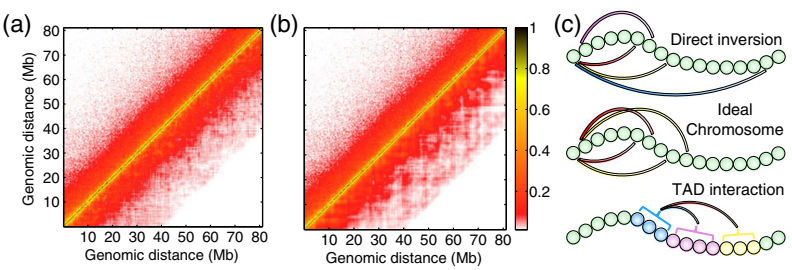

FIG. 1. Comparison between contact probabilities obtained from experiment and simulation. (a),(b) Contact probability maps for the mitotic chromosome as determined in $\mathrm{Hi}-\mathrm{C}$ experiments [7] (upper triangles) and as sampled in simulations based on the maximum entropy information theoretic energy landscape (lower triangles) from direct inversion (a) and from the TAD-augmented ideal chromosome model (b). (c) Schematic representation for the Hamiltonian for direct inversion (top), for the ideal chromosome model (middle), and for the sequence dependent interactions among topologically associating domains (bottom). The chromosome is drawn as beads on a string, and the interactions among genomic loci are drawn as curved lines, with different colors indicating varying strengths. microscope. Figure 2(a) shows that the simulated structures naturally exhibit cylindrical shapes, like those seen by light microscopy. This observed anisotropy indicates the metaphase chromosome must break rotational symmetry and is not an isotropic fluid or simple collapsed polymer phase. To quantitatively characterize the geometry of the simulated chromosome ensemble, we determined the statistics of the extension lengths of each structure along its three principal axes, as shown in the inset of Fig. 2(a). The distribution for the longest axes peaks at a value twice that for the shorter two, which are comparable, indicating the typical cylindrical shape of the mitotic chromosome. In contrast, when a landscape for the interphase chromosome is constructed using $\mathrm{Hi}-\mathrm{C}$ data synchronized at the G1 phase, the resulting structures are more nearly spherical with principal axis ratios typical of an isotropic weakly confined polymer (see Fig. S2(f) [17]).

Figure 2(b) shows a representative simulated structure of the mitotic chromosome. The bead color traverses from blue to cyan to yellow and to red along the sequence. The structure shown on the left at full resolution suggests the presence of a helical twist along the sequence, which is easier to visualize in the coarsened representation shown on the right for segments $5 \mathrm{Mb}$ in length, corresponding to a 50 times coarser description. The global helixlike structure clearly signals the presence of cholesteric liquid crystalline ordering in the mitotic chromosome. Helical conformations have indeed been observed experimentally for the mitotic chromosome [27], and sister chromatids produced on cell division are reported to break chiral symmetry, each having an opposite helical handedness.

To characterize quantitatively the magnitude of the helical twist, we introduce a local collective chiral variable $\psi(i)$ for a genomic locus $i$ along the chromosome as illustrated in the inset of Fig. 3(a), modeled on chirality
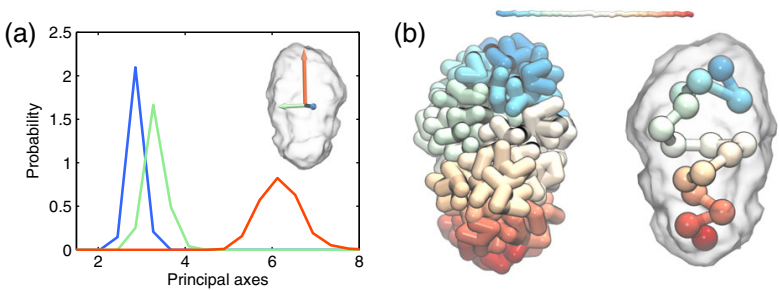

FIG. 2. Structural characterization of the simulated mitotic chromosome conformations. (a) Probability distributions for the extension lengths along the three principal axes, an illustration of which is provided in the inset. A sampled chromosome structure is shown in the transparent surface in the inset along with the principal axes indicated as arrows. (b) A representative structure from the simulated ensemble shown at full resolution (left) and at coarsened resolution (right) illustrates the presence of a twist along the sequence. For the coarsened picture, the full resolution chromosome is shown in the transparent surface. A color bar that indicates the color variation along the sequence is provided at the top. 
(a)

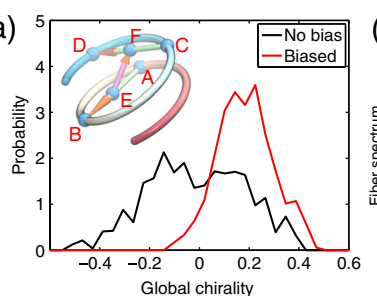

(c)

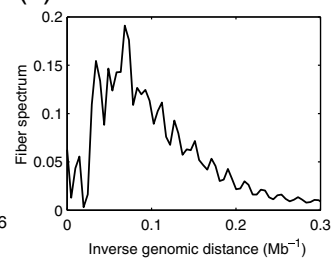

(b)

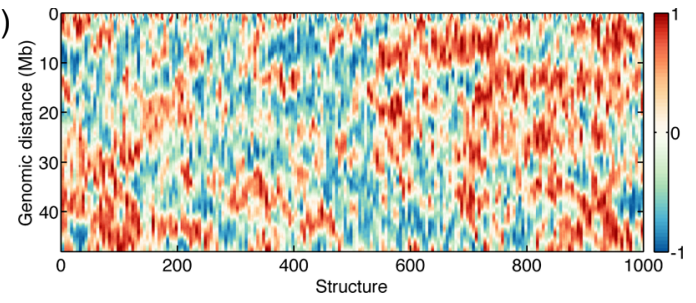

FIG. 3. Quantitative characterization of twist motifs in chromosome structures. (a) The probability distributions of the global chirality for the ensemble of structures from chromosome models without torsional bias (black) compared with that with torsional bias (red). An illustration defining of the collective variable $\psi$ that measures the handedness of a given chiral structure is shown in the inset. A twisted filament is drawn and colored along its sequence from red to blue, and for this filament $\psi$ can be defined with the three vectors highlighted as arrows. (b) Density plots of $\psi$ along the genomic sequence for different chromosome structures from the simulated ensemble. (c) Fiber spectrum of the metaphase chromosome structures simulated with the presence of weak torsional biases.

variables introduced for peptides and nucleic acid chains $[28,29] . \psi(i)=\overrightarrow{E F} \cdot(\overrightarrow{C D} \times \overrightarrow{A B}) /(|\overrightarrow{E F}| \cdot|\overrightarrow{C D}| \cdot|\overrightarrow{A B}|)$ and distinguishes right-handed from left-handed twists with positive and negative values, respectively. The vectors $\overrightarrow{A B}$, $\overrightarrow{C D}$, and $\overrightarrow{E F}$ are chosen based on the genomic position of locus $i$ to detect the long-range helicity illustrated in Fig. 2(b) occurring over tens of megabases [17].

Figure 3(b) plots the $\psi(i)$ for the ensemble of simulated chromosome structures with red corresponding to a righthanded twist and blue to a left-handed twist. Each column in this figure corresponds to a single sampled chromosome structure. A full chirally ordered structure would appear as a uniformly red or blue column. Most columns, however, break into blocks of red and blue segments, indicating that global ordering is often kinetically or thermodynamically prevented from going to completion. As shown in Fig. S1(f) [17], the genomic distance correlation length for the chirality variable $\psi(i)$ is shorter than the entire chromosome. So the probability distribution of $\psi(i)$ averaged over the entire chromosome has only a single peak near zero [Fig. 3(a), black], due to the cancellation from a mixture of right-handed (positive) and left-handed (negative) twists in still rather long segments.

Clearly, the chromosome has a high susceptibility to chiral order. Since the chiral domain boundaries can at best diffuse, we believe kinetics has prevented observing complete global symmetry breaking. To test for global ordering, we then explicitly but weakly break the chiral symmetry by applying a weak torsional bias locally to the chain. As shown in Fig. S1(e) [17], the distribution of torsional dihedral angles without the weak bias exhibits two peaks at both positive and negative values, which arise from the right-handed and left-handed segments in the structure, respectively. In contrast, as shown in Fig. S3 [17], when weak biases are introduced, reoptimization leads to a unimodal distribution. The resulting structures now exhibit a global twist, with the chirality distribution peaking around 0.2 [Fig. 3(a), red line]. Remarkably, the contact maps obtained from simulations incorporating the weak local dihedral bias are equivalent in quality to those found without introducing any local chirality bias (see Fig. S3 [17]).

The Hi-C contact map data by themselves are not sufficient to distinguish structures with global twist from an ensemble of structures in which the chromosome is broken into long segments with differing chirality. Since the Hi-C data contain only pairwise information, mirror image conformations are energetically degenerate. The presence of a global chiral twist of the mitotic chromosome structure is consistent with what is postulated in hierarchical models, in which the chromosome folds successively by forming fibers of fibers [2]. Hierarchical models incorporate multiple layers of twisting and fibril structures at several different length scales. The chiral twist collective variable probes structure at the $25 \mathrm{Mb}$ scale. The fiber spectrum that we introduced in our previous study of the interphase chromosome gives evidence for structures at finer scales [4], as shown in Fig. 3(c). The fiber spectrum is the sequence Fourier transform of an orientation order parameter defined by the scalar product of two displacement vectors along the genomic sequence [17], and exhibits peaks at frequencies (inverse sequence separation) that correspond to the period of the twist.

How does the system break the symmetry, and transition from the isotropic interphase configuration to the cylindrical and twisted chiral metaphase structures? To reveal the underlying physical mechanism of this transition, we have also built simplified chromosome models that take into account two of the most prominent features of the system from our previous study of the interphase chromosome. First, the model includes a homogeneous ideal chromosome interaction, which represents an effective homopolymer with a pairwise contact potential, whose strength depends only on the sequence separation of the two genomic loci, not on their specific locations. This homogenized model encodes only the sequence translation invariant features of the DNA along with its protein packaging. We previously found that a hierarchically organized chromosome with local liquid crystalline features and orientational ordering can arise from a generic ideal chromosome model [4]. An illustration of the ideal 
chromosome model is provided in the middle panel of Fig. 1(c). Second, sequence specific interactions among topologically associating domains with varying chemical compositions are introduced into this model. This term is motivated by the observation that the interphase chromosome displays clear deviations from the best equivalent homopolymer landscape, exhibiting clumps or topologically associating domains that are of size $\sim 1 \mathrm{Mb}$ along the genomic sequence [30], as highlighted in the interphase Hi-C map shown in Fig. S5 [17]. Topologically associating domains have different histone modifications [31,32], and may play crucial roles in gene regulation [33]. Establishing strict boundaries between topologically associating domains necessitates there being deviations from the homogenized ideal chromosome model. These energetic inhomogeneities can pin the motions of defects in helical or chiral ordering [34].

The Hamiltonian for this topologically associating domain (TAD)-augmented ideal chromosome model is

$$
\begin{aligned}
U_{\mathrm{TI}}(r)= & U(r)+\sum_{i j} \alpha_{\text {ideal }}(|j-i|) f\left(r_{i j}\right) \\
& +\lambda \sum_{A, B} \alpha_{A B} \sum_{i \in A} \sum_{j \in B} f\left(r_{i j}\right) .
\end{aligned}
$$

The first term is the same homopolymer potential as in Eq. (1). The second term corresponds to the homogeneous ideal chromosome contact potential, whose strength depends only on the sequence separation $|j-i|$ and is therefore sequence translation invariant. The final term explicitly breaks sequence translation invariance and describes sequence specific interactions at the level of the topologically associating domains found in the interphase, as illustrated in the bottom panel of Fig. 1(c). All the genomic loci in a given topologically associating domain $A$ are treated equally and when interacting with loci from another domain $B$ they share the same contact potential $\alpha_{A B}$. The simplification of treating all the genomic segments within a topologically associating domain equally is motivated by the observation that loci within a domain exhibit correlated histone modifications. As shown in Refs. [31,32], loci within a domain further exhibit nearly identical long-range contact patterns and often are in the same chromatin state. The prefactor $\lambda$ of the third term is introduced for studying the consequences of the pinning terms, and is set to 1 during the iterative fitting of the experimental contact map.

Again we use the maximum entropy scheme to find the strength of $\alpha_{\text {ideal }}$ and $\alpha_{A B}$ for both interphase and metaphase chromosomes (see the Supplemental Material [17] for details). For the metaphase chromosome, we also further incorporated very weak dihedral biases as in the direct inversion to encourage global twisting. Figures 1(b) and S6 [17] together demonstrate that the TAD-augmented ideal chromosome model of the mitotic chromosome again reproduces the contact map and the power law scaling of the contact probability. Just as for the direct inversion, chromosome conformations produced with $U_{\mathrm{TI}}(r)$ adopt cylindrical shapes with global twisting. As shown in Fig. S6(f) [17], the energies for the TAD-augmented ideal chromosome model are significantly correlated to those from the Hamiltonian obtained using direct inversion when sampled over the structural ensemble.

Comparing the interphase and metaphase potentials suggests a possible mechanism for chromosome condensation. As shown in Fig. S8(a) [17], the main difference between the interphase and metaphase ideal chromosome contact potentials $\alpha_{\text {ideal }}(|j-i|)$ is that the metaphase interactions are consistently stronger at both short- and long-range sequence separation. Even when the domain inducing interactions are neglected, the resulting ideal chromosome landscape including only the first two terms in Eq. (3) gives rise to condensed and twisted structures as shown by the chirality variable and the length of the principal axes in Fig. S8 [17]. In the main, the condensation of the mitotic chromosome can be understood through the emergence of a strong ideal chromosome potential that naturally leads to hierarchical fiber ordering.

To further understand the role of the "random" fields that arise from interactions among topologically associating domains that form in the interphase chromosome, we compute the phase diagram for $U_{\mathrm{TI}}(r)$ as a function of both the information temperature $T$ and the inhomogeneity prefactor $\lambda$. We note the information temperature $T$ has a clear physical meaning and is related to the notion of an effective temperature that provides an effective equilibrium description for nonequilibrium systems [35,36]. Twodimensional plots of the global chiral ordering and the ratio of the lengths for the longest and shortest principal axes are presented in Figs. 4(a) and 4(b), respectively. While the homogeneous ideal chromosome contact potential promotes both hierarchical fiber and chiral ordering, the interactions among topologically associating domains destroy chiral order. We note the changes occurring here may either be sharp transitions or crossovers. Chiral ordering is also lost with increasing temperature $T$ through the unwrapping of the helical twist. This is evidenced by the increase of chromosome length shown in Fig. 4(b). Examples of chromosome conformations at various temperatures for $\lambda=1$ are provided in Fig. 4(c). On the other hand, the chromosome still remains condensed at large inhomogeneity strength $\lambda$, and the chiral ordering is lost through the formation of defects in the chiral structure.

Chromosome macroscale organization arises from a balance between the ideal chromosome contact potential that promotes helical, chiral, and cylindrical ordering and the interactions among topologically associating domains that encourage defects in such order. The coupling between shape changes and the chiral and liquid crystalline ordering is strong. We can see this clearly through equilibrium 

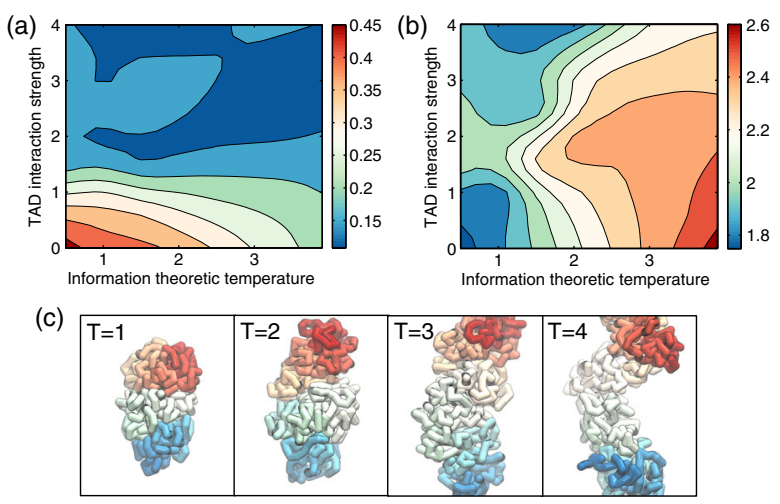

FIG. 4. Phase diagrams for the TAD-augmented ideal chromosome model of the mitotic chromosome. (a),(b) The global chirality (a) and ratio of the largest and smallest extension length along the principal axes (b) are plotted as a function of temperature $T$ and inhomogeneity strength $\lambda$. (c) Representative chromosome structures at varying temperature $T$ for $\lambda=1$ that illustrate the loss of chirality as the helical twist unwinds.

simulations using the homogenized ideal chromosome model without torsional bias at a high information temperature $T=1.5$. The combination of high temperature and the absence of defect pinning interactions allows full equilibration. The chirality transition in this ideal model is accompanied by the structural extension of the chromosome. This can be seen in Fig. 5, which displays the free energy surface as a simultaneous function of the longest principal axis length of the chromosome and its global chirality. An example reactive trajectory shown in green plotted on top of this free energy surface shows that extending the chromosome can switch the chirality.

It is worth pointing out that the interactions of our effective chromosome models are derived from Hi-C contact probabilities, which can not only be affected by intrinsic physicochemical interactions of the DNA, but also the numerous molecular players in the nucleus environment. Therefore, we cannot rule out that the broken symmetry could possibly be caused by an anisotropic

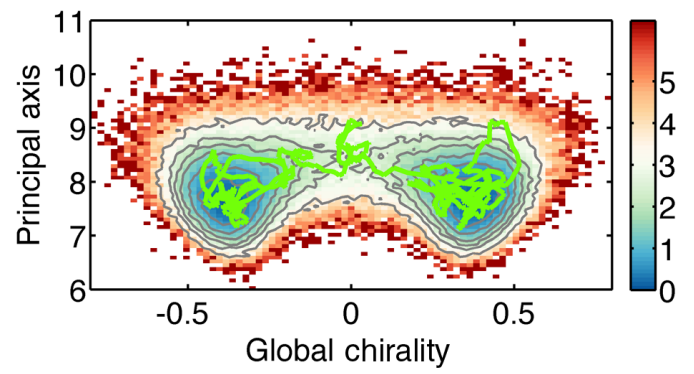

FIG. 5. Coupling between chromosome shape changes and chiral ordering. The free energy as a function of the global chirality and the length of the longest principal axis is shown as a surface plot. Contours of constant free energy are drawn as gray lines. An example trajectory that connects the two chiral conformations is plotted in green. environment rather than only microscopic interactions within the chromosome itself, and the chiral order in chromosomes may be set up by the cellular machinery that locates the chromosome within the cell.

This work was supported by the Center for Theoretical Biological Physics sponsored by the NSF (Grants No. PHY-1308264 and No. PHY-1427654). P. G. W. acknowledges financial support by the D. R. BullardWelch Chair (Grant No. C-0016) at Rice University.

* Corresponding author. pwolynes@rice.edu

[1] J. F. Marko, Chromosome research 16, 469 (2008).

[2] N. Kireeva, M. Lakonishok, I. Kireev, T. Hirano, and A. S. Belmont, J. Cell Biol. 166, 775 (2004).

[3] K. Maeshima and U. K. Laemmli, Dev. Cell 4, 467 (2003).

[4] B. Zhang and P. G. Wolynes, Proc. Natl. Acad. Sci. U.S.A. 112, 6062 (2015).

[5] H. Kang, Y.-G. Yoon, D. Thirumalai, and C. Hyeon, Phys. Rev. Lett. 115, 198102 (2015).

[6] E. Lieberman-Aiden, N. L. van Berkum, L. Williams, M. Imakaev, T. Ragoczy, A. Telling, I. Amit, B. R. Lajoie, P. J. Sabo, M. O. Dorschner, R. Sandstrom, B. Bernstein, M. A. Bender, M. Groudine, A. Gnirke, J. Stamatoyannopoulos, L. A. Mirny, E. S. Lander, and J. Dekker, Science 326, 289 (2009)

[7] N. Naumova, M. Imakaev, G. Fudenberg, Y. Zhan, B. R. Lajoie, L. A. Mirny, and J. Dekker, Science 342, 948 (2013).

[8] M. Di Stefano, A. Rosa, V. Belcastro, D. di Bernardo, and C. Micheletti, PLoS Comput. Biol. 9, e1003019 (2013).

[9] M. Bohn and D.W. Heermann, PLoS One 5, e12218 (2010).

[10] M. Barbieri, M. Chotalia, J. Fraser, L. M. Lavitas, J. Dostie, A. Pombo, and M. Nicodemi, Proc. Natl. Acad. Sci. U.S.A. 109, 16173 (2012).

[11] G. Gürsoy, Y. Xu, A. L. Kenter, and J. Liang, Nucleic Acids Res. 42, 8223 (2014).

[12] F. Benedetti, J. Dorier, Y. Burnier, and A. Stasiak, Nucleic Acids Res. 42, 2848 (2014).

[13] G. Tiana, A. Amitai, T. Pollex, T. Piolot, D. Holcman, E. Heard, and L. Giorgetti, Biophys. J. 110, 1234 (2016).

[14] R. R. Cheng, F. Morcos, H. Levine, and J. N. Onuchic, Proc. Natl. Acad. Sci. U.S.A. 111, E563 (2014).

[15] T. R. Lezon, J. R. Banavar, M. Cieplak, A. Maritan, and N. V. Fedoroff, Proc. Natl. Acad. Sci. U.S.A. 103, 19033 (2006).

[16] E. Schneidman, M. J. Berry, R. Segev, and W. Bialek, Nature (London) 440, 1007 (2006).

[17] See Supplemental Material at http://link.aps.org/ supplemental/10.1103/PhysRevLett.116.248101, which includes Refs. [18-25], for details.

[18] M. Imakaev, G. Fudenberg, R. P. McCord, N. Naumova, A. Goloborodko, B. R. Lajoie, J. Dekker, and L. A. Mirny, Nat. Methods 9, 999 (2012).

[19] S. Wang and P. G. Wolynes, J. Chem. Phys. 136, 145102 (2012). 
[20] A. Rosa and R. Everaers, PLoS Comput. Biol. 4, e1000153 (2008).

[21] C. Munkel and J. Langowski, Phys. Rev. E 57, 5888 (1998).

[22] K. Bystricky, P. Heun, L. Gehlen, J. Langowski, and S. M. Gasser, Proc. Natl. Acad. Sci. U.S.A. 101, 16495 (2004).

[23] A. Bolzer, G. Kreth, I. Solovei, D. Koehler, K. Saracoglu, C. Fauth, S. Müller, R. Eils, C. Cremer, M. R. Speicher, and T. Cremer, PLoS Biol. 3, e157 (2005).

[24] G. Fiorin, M. L. Klein, and J. Henin, Mol. Phys. 111, 3345 (2013).

[25] D. Bau and M. A. Marti-Renom, Methods 58, 300 (2012).

[26] M. P. Eastwood, C. Hardin, Z. Luthey-Schulten, and P. G. Wolynes, J. Chem. Phys. 117, 4602 (2002).

[27] E. B. de la Tour and U. K. Laemmli, Cell 55, 937 (1988).

[28] M. Moradi, V. Babin, C. Roland, T. A. Darden, and C. Sagui, Proc. Natl. Acad. Sci. U.S.A. 106, 20746 (2009).
[29] M. Moradi, V. Babin, C. Roland, and C. Sagui, Nucleic Acids Res. 41, 33 (2013).

[30] J. R. Dixon, S. Selvaraj, F. Yue, A. Kim, Y. Li, Y. Shen, M. Hu, J. S. Liu, and B. Ren, Nature (London) 485, 376 (2012).

[31] S. P. Rao, M. Huntley, N. Durand, E. Stamenova, I. Bochkov, J. Robinson, A. Sanborn, I. Machol, A. Omer, E. Lander, and E. Aiden, Cell 159, 1665 (2014).

[32] T. Sexton, E. Yaffe, E. Kenigsberg, F. Bantignies, B. Leblanc, M. Hoichman, H. Parrinello, A. Tanay, and G. Cavalli, Cell 148, 458 (2012).

[33] B. Zhang and P. G. Wolynes, Proc. Natl. Acad. Sci. U.S.A. 111, 10185 (2014).

[34] Z. Luthey-Schulten, B. E. Ramirez, and P. G. Wolynes, J. Phys. Chem. 99, 2177 (1995).

[35] S. Wang and P. G. Wolynes, J. Chem. Phys. 135, 051101 (2011).

[36] L. F. Cugliandolo, J. Phys. A 44, 483001 (2011). 